\title{
The Use of Fuzzy Cognitive Maps for the Analysis of Structure of Social and Economic System for the Purpose of Its Sustainable Development
}

\author{
Larisa A. Ginis \\ Southern Federal Iniversity, Russian Federation \\ Email: gla@sfedu.ru
}

\section{Doi:10.5901/mjss.2015.v6n3s5p113}

\begin{abstract}
The paper aimed to consideration of a problem of modeling of complex systems. The use of fuzzy cognitive maps for modeling and the analysis of functioning of complex systems is offered. Features of difficult system on the example of social and economic system are analyzed to work The author seeks to track process of change of views and approaches on modeling of a sustainable development of social and economic systems. The problem is formulated on the basis of synthesis of concepts of the theory of systems and the theory of decision-making, the theory of fuzzy graphs and the theory of hierarchical multilevel systems that expands possibilities of the accounting of uncertainty various nature, including risk of a human factor. The idea locates and the concept of an fuzzy cognitive map as fuzzy directed graph unlike known examples of fuzzy cognitive maps is entered. In this work the concept of fuzzy bases of the fuzzy first way focused the column is used, approach to their application for the analysis of fuzzy cognitive maps is considered. The special attention is paid to the description of an example illustrating possibilities of modeling of a trajectory of a sustainable development of social and economic systems by means of fuzzy cognitive maps in the form of fuzzy directed graph. The example of work of the Matrix program developed earlier realizing known algorithms of stay is given: fuzzy ways between concepts; approachibility matrixes; definitions of a set of fuzzy bases, connectivity in this article. This all is important for forecasting of sustainable developing social and economic system.
\end{abstract}

Keywords: complex system, fuzzy cognitive map, sustainable development, social and economic system

\section{Introduction}

Relevance of the presented work is proved by the requirement of the accounting of a trajectory of a sustainable development of the social and economic systems (SES) which increased in the XXI century at adoption of administrative decisions (Slesarev \& Yanovskiy, 2014; Tretyakova, 2013; Yu \& Wu, 2014). That is why research of structural stability of SES will be one of actual problems in the field of modeling of complex systems which typical representative is the social and economic system.

The social and economic system as a complex system, on micro and at the macro levels possesses such property as complexity (Morales-Matamoros et al., 2010).

Complex systems exist as set of objects on production, in the social sphere, in economy, in scientific and technical development. The main distinctive features of complex systems are numerous and various by the nature and type of communication between separately existing elements of system. Moreover, these communications are dynamic by the nature that complicates a task modeling of such system and even more increases relevance. Communications between elements of complex system are always characterized by a certain order, internal properties, an orientation on performance of functions of system. Such features of this concrete system are accepted to call structure.

It is obvious that in structure it is inherent some general regularities, and it can separately be studied, irrespective of the concrete contents and purpose of complex system. It is also possible to speak about application in system of various knowledge and technical means, modeling and simplification, the centralized use of information.

High sensitivity to different revolting influences of environment is one more important feature of complex system. This characteristic and remains today thoroughly not studied, and therefore also actual for research if we want to understand how to construct model of system for development of reasonable administrative decisions for the purpose of its sustainable and safe development. Similar questions are often brought up in modern researches (Gamage et al., 2013; Kalmar-Nagy \& Stanciulescu, 2014; Xiong \& Han Zhao, 2014). Studying of development of difficult systems - one of important modern tasks, which was more studied only at a stage of supervision over such systems.

Relevance of research is caused, including such factors as accumulation of new knowledge and data on modeling of difficult systems for the last 10-15 years, and as a result, a new problem definition, for example as in (Bozhenyuk \& 
Ginis, 2014b; Bozhenyuk \& Rozenberg, 2012a; Miller \& Scott, 2007; Khokher et al., 2013).

\section{Theoretical Analyses}

It is convenient to represent structure of system in the form of the graphic scheme consisting of tops, and lines connecting them - communications that is described in (Luqman et al., 2013). Such schemes are called structural. As such block diagram in this paper it is offered to use the fuzzy cognitive map (FCM) other than cognitive maps of type: Silov's fuzzy cognitive maps (Silov 1995); Rules Based Fuzzy Cognitive Maps (Carvalho 2012); Generalized Rule Based Fuzzy Cognitive Maps (Fedulov \& Borisov, 2004); Relational Fuzzy Cognitive Maps and Fuzzy Relational Maps (Fedulov 2005), Neutrosophic Relational Maps (Kandasamy et al., 2003), dynamic cognitive of networks (Miao et al., 2010).

That fact is the distinctive reason of application of FCMs in the form of a fuzzy graph that mathematical the device of the theory of fuzzy graph allows to trace and investigate unequal communications between concepts when influence in one of the directions has much bigger impact on an element, than in other one.

Besides, for the last 20 years the device of the theory of fuzzy graph developed rather roughly, settled and finds broad application in applied tasks (Vaishnaw \& Sharma, 2012). And also it is well realized in languages programming of high level, allowing to develop programs for finding of the decision for the acceptable time in the automated mode (Bozhenyuk \& Gerasimenko, 2014a).

So, we will understand as an fuzzy cognitive map the fuzzy directed graph who is set by pair of sets $\widetilde{G}=(X, \widetilde{U})$ in which $X=\left\{x_{i}\right\}, i \in l=\{1,2, \ldots, n\}-$ is accepted an crisp set of concepts as which the factors, most essential to the considered problem, get out, and $\widetilde{U}=\left\{\left\langle\mu_{u}<x_{i}, X_{k}\right\rangle /\left\langle x_{i}, X_{k}\right\rangle>\right\}$ is a fuzzy set of edges (or arcs), where $\left\langle x_{i}, X_{k}\right\rangle \in X^{2}$, and $\left.\mu_{u}<x_{i}, X_{k}\right\rangle$ is a degree of membership oriented edge $\left\langle x_{i}, x_{k}\right\rangle$ to fuzzy set of directed edges $\widetilde{U}$.

Also FCM can be given in the form $\widetilde{G}=(X, \widetilde{\Gamma})$, where $X=\left\{x_{i}\right\}, i \in l=\{1,2, \ldots, n\}$, and $\widetilde{\Gamma}$ - fuzzy valued mapping of the vertex set $X$ into itself, ie, $\widetilde{\Gamma}: X \rightarrow X$, which is given in the form of fuzzy images of the elements $x \in \mathrm{X}$, i.e, $\widetilde{\Gamma}$ $\left(x_{i}\right)=\left\{\left\langle\mu r\left(x_{j}\right) / x_{j}\right)\right\}, x_{j} \in \Gamma\left(x_{i}\right)$, here $\Gamma\left(x_{i}\right)$ is a crisp set of images of the vertices $x_{i} \in X$ (Bershtein \& Bozhenyuk, 2005, Mordeson 2000).

The fuzzy cognitive map can be set and in the form of an fuzzy directed graphs of the second way $\widetilde{G}=(\widetilde{X}, \widetilde{U})$, where $\tilde{X}$ - the set of concepts which is an fuzzy set in some universal great number of $X$, i.e. $\tilde{X}=\left\{\left\langle\mu_{x}(x) / x>\right\}, x \in X\right.$, $|X|=n$, with function of accessory $\mu x: X \rightarrow[0,1]$, and $\widetilde{U}$ - the fuzzy set of the focused edges (or arches) decides as $\widetilde{U}=\left\{\left\langle\mu_{u}\left\langle X_{i}, X_{k}\right\rangle /\left\langle X_{i}, X_{k}\right\rangle>\right\},\left\langle X_{i}, X_{k}\right\rangle \in X^{2}\right.$, on function of accessory $\mu v: X^{2} \rightarrow[0,1]$ (Bozhenyuk 2012b). We will note that fuzzy directed graphs of the 2 nd sort if necessary it is possible to transform unambiguously in fuzzy directed graphs of the 1st sort, as well as on the contrary, but in this case we will receive infinitely many fuzzy graphs of the second sort that isn't optimum.

FCMs can act as concepts (tops, objects, and judgments), any objects of political, economic, social, ecological system or their set, for example, socio-economic indexes, political decisions and their effects, investments into health care and a demography, the purposes and necessary means of their achievement, etc. The revealed connections between tops and its importance will be fuzzy arches.

For studying of SES often use reception of decomposition which essence is simplification of the system too difficult for consideration entirely. Such simplification can:

a) actually to lead to replacement of system by some other, in some sense corresponding to the initial. As a rule, it becomes input of hypotheses of rejection or weakening of separate communications of system;

b) completely to correspond to initial system and thus to facilitate work with it - such decomposition it is accepted to call strict or isomorphic that demands special methods of coordination and coordination of consideration of parts.

The use of the offered FCMs type allows to carry out such simplification and as on the way a), and on the way b) since the indistinct organ by the nature is easily subject to changes and realizes property of isomorphism.

It is necessary to be guided by the following fundamental principles at creation of model which have to be put in any SES model: principle of the accounting of risk of a human factor; principle of reflection of interaction of the person and environment; the principle of controllability of model, the principle of feasibility of model in the dialogue mode between the researcher and the computer. And also some principles of systemacity: principle of unity and communication; the principle of hierarchy offering introduction of hierarchy of elements and/or their ranging; the principle of functionality dictating joint consideration of structure and function with a function priority over structure; the principle of 
development obliges to consider convertibility of system, its ability to development, expansion, replacement of elements, accumulation of information; principle of uncertainty: the accounting of uncertainty and accidents in system; the principle of sensitivity says that intervention in system has to be coordinated with the level of its reaction to intervention; principle of convolution: information and managing directors influence curl up (are integrated, generalized) at the movement from below up hierarchical levels.

Thus, we will understand the way of its existence fixing quite certain priorities and interrelations of its elements as structure of system. For each system it is possible to construct some types of structures.

Considering the called features and the designated list of the principles, the idea hierarchical is the basis for modeling of SES or the so-called stratified description of system (Haddad et al., 2006, Mesarovic et al., 1970), the developed model is partially described in (Bozhenyuk \& Ginis, 2015).

\section{The Research Example}

Structural stability of difficult system is directly interconnected with property of robustness of system (Wagner, 2005).

From the point of view of system approach - it is accepted to understand robustness of the damaged system to adapt to new changed and, as a rule, to unforeseen situations, to resist to harmful effects as robustness, carrying out thus in whole or in part the criterion function, due to corresponding change of structure and behavior of system. Also robustness can be understood as firmness of system, in our case of structure. Depending on degree of complexity of structure property of survivability is shown as difficult integrated quality of system and, respectively, is quantitatively estimated by indicators of stability, durability, reliability, adaptability, fault tolerance, a noise stability, etc.

From the point of view of the theory of directed graphs in FCMs under robustness we will understand degree of strong connectivity of directed graphs (Bozhenyuk \& Ginis, 2013).

We accept that all SES which are subjects to modeling are steady or quasi-stable. It is offered to define stability of structure of SES by definition of fuzzy base and robustness of FCM (Bozhenyuk et al., 2014c) for the purpose of definition of a trajectory of sustainable and safe development of the operated system.

The most important components of SES are innovative activity, developmental development, such problems of economy as environmental problems of development territorial and production (or regional) complexes of the Russian Federation, demographic and food security and a number of essentially new elements, for example as in (Papchenko, 2004).

The sustainable development of regional SES is defined by the following set of components of safe development (see Figure 1).

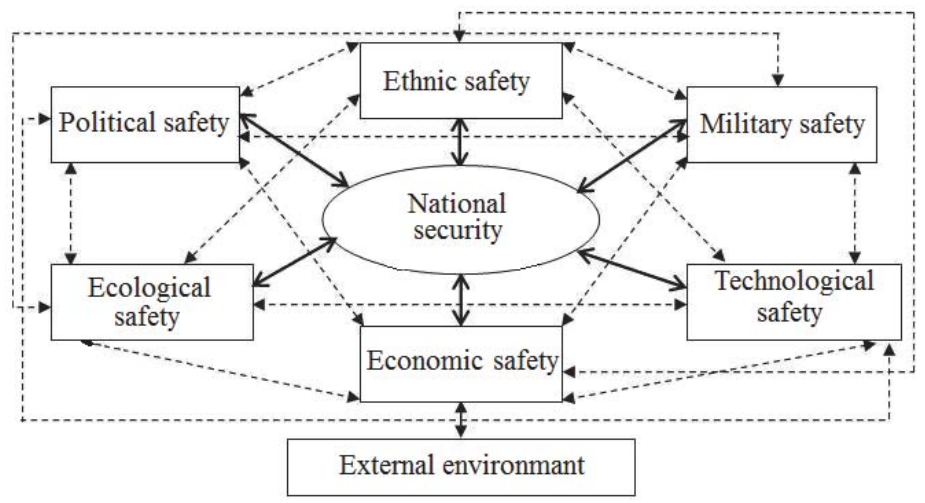

Figure 1. The cognitive model of structure of national security of SES

We will enter the following designations: X1 - political safety, X2 - ethnic safety, X3 - military safety, X4 - technological safety, X5 - economic safety, X6 - ecological safety. Then FCM at one level of hierarchy assumes the following air (see Figure 2). 


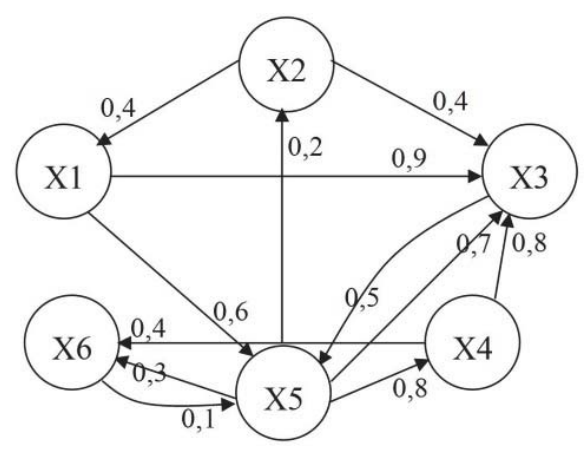

Figure 2. FCM at one level

We will present represented in figure 2 FCM in the form of incidence matrix (see Figure 3).

$$
W=\left(\begin{array}{cccccc}
0 & 0 & 0,9 & 0 & 0,6 & 0 \\
0,4 & 0 & 0,4 & 0 & 0 & 0 \\
0 & 0 & 0 & 0 & 0,5 & 0 \\
0 & 0 & 0,8 & 0 & 0 & 0,4 \\
0 & 0,2 & 0,7 & 0,8 & 0 & 0,3 \\
0 & 0 & 0 & 0 & 0,1 & 0
\end{array}\right)
$$

Figure 3. Incidence matrix

\section{Results of Research}

We will find all fuzzy ways between concepts, we will construct the approachability matrix and we will define a set of fuzzy bases for what we will use specially developed Matrix (Bozhenyuk \& Gerasimenko, 2014a) program (see Figure 4).

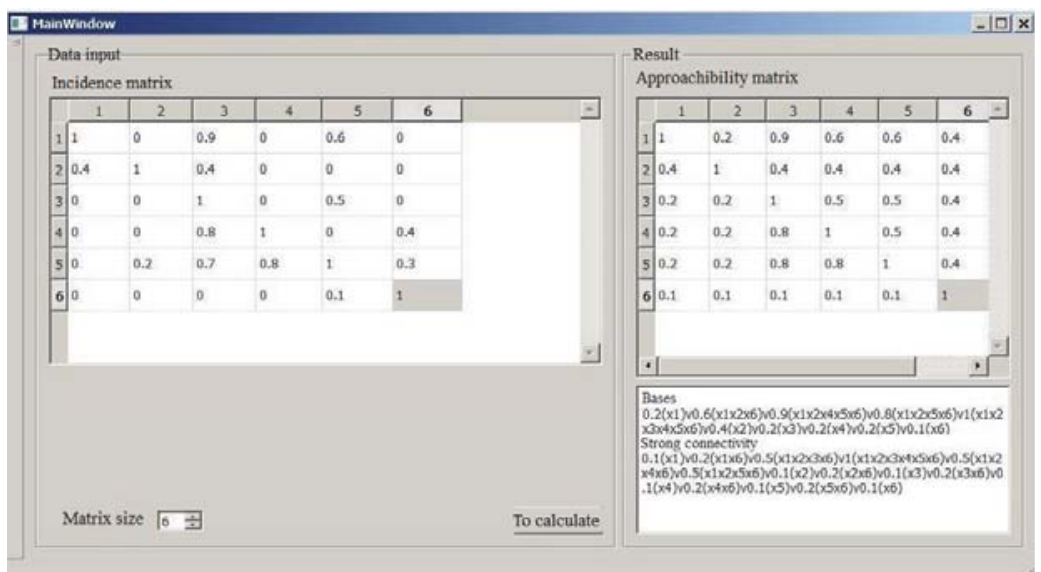

Figure 4. Result of calculation in the program

Thus, for the described example, FCM has the following fuzzy bases: $B_{1}=\left\{x_{1}, x_{2}, x_{3}, x_{4}, x_{5}, x_{6}\right\}, B_{0,9}=\left\{x_{1}, x_{2}, x_{4}, x_{5}, x_{6}\right\}$, $B_{0,8}=\left\{x_{1}, x_{2}, x_{5}, x_{6}\right\}, B_{0,6}=\left\{x_{1}, x_{2}, x_{6}\right\}, B_{0,4}=\left\{x_{2}\right\}, B_{0,2}^{\prime}=\left\{x_{3}\right\}, B^{\prime \prime}{ }_{0,2}=\left\{x_{4}\right\}, B^{\prime \prime \prime}{ }_{0,2}=\left\{x_{5}\right\}, B^{\prime \prime} V_{0,2}=\left\{x_{1}\right\}, B_{0,1}=\left\{x_{6}\right\}$, and the fuzzy set of bases is defined as $\left.\left.\widetilde{B}_{X}=\{\langle 1 / 6\rangle,<0,9 / 5\rangle,<0,8 / 4>,<0,6 / 3\right\rangle,<0,4 / 1>\right\}$. The given fuzzy set of bases allows to draw a conclusion that in the considered structure there is one concept from which all others are achievable with degree 
0,4 ; there are three concepts from which all others are achievable with degree 0,6 ; there are four concepts from which all others are achievable with degree 0,8 ; also there are a set of five concepts from which all others are achievable with degree 0,9 .

The analysis of the found set of fuzzy bases shows that in structure of system there is one concept, and it not only, at the pessimistic forecast in which it is possible to invest small resources and it will give a positive effect in development of all components of system. I.e. making active the chosen concept, and with small expenses, it is possible to achieve a certain improvement of a state in all other concepts of SES. This speaks about rather steady structure of model.

We will define degree of robustness of FCM. Analyzing the received set of strong connectivity (see Figure 4), we come to a conclusion that degree of robustness of our HKK=0,1, i.e. between two any concepts of FCM exists an fuzzy way with a conjunctive durability not less than 0,1 and it is obviously not a high rate. However, such value can be interpreted so: in this model dynamics of changes and interactions is high.

In total, the carried-out analysis allows to draw a conclusion that structural stability of system low, but there are concepts which activization even in the pessimistic forecast leads to a steady positive effect in development of all SES.

\section{Conclusion}

The modern social and economic system is characterized as the complex system described by set of qualitative and quantitative variables and factors. The offered approach with application of fuzzy cognitive maps in the form of fuzzy directed graphs will allow to construct steady structure of the SES model for the purpose of adoption of administrative decisions on its safe development. And the developed program Matrix simplifies work with FCM, irrespective of amount of interrelations and elements of system.

Distinctive feature of the presented paper is possibility of the accounting of a human factor when developing models for the purpose of active influence of the operated system on management process for support of adoption of optimum and reasonable administrative decisions.

\section{Acknowledgement}

This work has been supported by the Russian Foundation for Basic Research, Project № 15-07-00185a.

\section{References}

Bershtein, L.S., \& Bozhenyuk, A.V. (2005). Fuzzy graphs and hypergraphs. Moscow: Scientific world PRESS.

Bozhenyuk, A.V., \& Gerasimenko, E. (2014a). Flows Finding in Networks in Fuzzy Conditions. Studies in Fuzziness and Soft Computing, $313,269-291$.

Bozhenyuk, A.V., \& Ginis, L.A. (2013). The use of fuzzy graph models for the analysis of complex systems. Control systems and information technologies, 51(1.1), 122-124.

Bozhenyuk, A.V., \& Ginis, L.A. (2014b). Modeling and analysis of complex systems on the basis of fuzzy graph models. Life Science Journal, 11 (7), 187-191.

Bozhenyuk, A.V., \& Ginis, L.A. (2015). Algorithmic support of system communication research in socio-economic system based on fuzzy graph models. Economy and management of control systems, 15 (1.1), 115-122.

Bozhenyuk, A.V., \& Rozenberg, I. (2012a). Allocation of service centers in the GIS with the largest vitality degree. Communications in Computer and Information Science, 298 (2), 98-106.

Bozhenyuk, A.V., Bozhenyuk, V.A. \& Ginis, L.A. (2014c). Features of algorithmic realization of automation of finding of fuzzy bases in fuzzy directed graph of the first way. Information technologies of modeling and control, 5(89), 389-395.

Bozhenyuk, A.V., Rozenberg, I.N., \& Yastrebinskaya, D. (2012b). Finding of survivability of fuzzy transport networks with application of geographic information systems. Moscow: Scientific world PRESS.

Carvalho, J.P. (2012). Rule based fuzzy cognitive maps in humanities, social sciences and economics. Studies in Fuzziness and Soft Computing, 273, 289-300.

Fedulov, A.S. (2005). Relational Fuzzy Cognitive Maps. Izvestiya RAN. Teoriya i sistemy upravleniya, 1, 120-133.

Fedulov, A.S., \& Borisov, V.V. (2004). Generalized Rule - Based Fuzzy Cognitive Maps. Neurocomputers: design and applications, 4, 321.

Gamage, G.B., Boyle, C., \& McDowall, R. (2013). The development of an integrated model for assessing sustainability of complex systems. International Journal of Sustainable Development, 16 (3/4), 297-309.

Haddad, W.M., Chellaboina, V., \& Nersesov, S. (2006). Impulsive and Hybrid Dynamical Systems: Stability, Dissipativity and Control. Princeton University Press.

Kalmar-Nagy, T., \& Stanciulescu, L. (2014). Can complex systems really be simulated. Applied Mathematics and Computation, 227, $199-211$. 
Kandasamy, W.B., \& Vasantha Smarandache, F. (2003). Fuzzy Cognitive Maps and Neutrosophic Cognitive Maps. Xiquan: Phoenix.

Khokher, M.R., Ghafoor, A., \& Siddiqui, A.M. (2013). Image segmentation using multilevel graph cuts and graph development using fuzzy rule-based system. IET Image Processing, 7 (3), 201-211.

Luqman, M.M., Ramel, JY., Llados, J., \& Brouard, T. (2013). Fuzzy multilevel graph embedding. Pattern recognition, 46 (2), $551-565$.

Mesarovic, M.D., Macko, D., \& Takahara, Y. (1970). Theory of hierarchical, multilevel systems. New York, London: Academic Press.

Miao, Y., Miao, C., Tao, X., Shen, Z., \& Liu, Z. (2010). Transformation of cognitive maps. IEEE Transactions on Fuzzy Systems, 18 (1), 114-124.

Miller, J.H., \& Scott, E. (2007). Complex Adaptive Systems: An Introduction to Computational Models of Social Life. Princeton University Press.

Morales-Matamoros, O., Tejeida-Padilla, R., \& Badillo-Pina, I. (2010). Fractal behavior of complex systems. Systems Research and Behavioral Science, 27(1), 71-86.

Mordeson, J.N. (2000). Fuzzy Graphs and Fuzzy Hypergraphs. Springer.

Papchenko, E.V. (2004). The problems of olfaktorny safety. Izvestiya SFedu. Engineering sciences, 8(43), 262-263.

Silov, V.B. (1995). Adoption of strategic decisions in fuzzy situation. Moscow: INPRO-RES.

Slesarev, D., \& Yanovskiy, V. (2014). Sustainable development of the regional social-economic system: An innovative dimension. Public Policy and Administration, 13 (1), 9-21.

Tretyakova, E.A. (2013). Evolution of research and evaluation methodology of sustainable development of social and economic systems. World Applied Sciences Journal, 25 (5), 756-759.

Vaishnaw, Y., \& Sharma, S. (2012). Some analoges results on fuzzy graphs. Int Jr. of Mathematics Sciences \& Applications, 2(2), 535539.

Wagner, A. (2005). Robustness and Evolvability in Living Systems. Princeton University Press.

Xiong, D.C., \& Han Zhao Y.H. (2014). Optimal robust decentralized control design for fuzzy complex systems. Journal of Intelligent and Fuzzy Systems, 26(1), 211-222.

Yu, Y.J., \& Wu, L. (2014). Sustainable development of the Dongting lake flood detention basin's economic and social based on the system dynamics. Advanced Materials Research, 962-965, 2249-2252. 